\title{
Downregulation of microRNA-199a-5p protects cardiomyocytes in cyanotic congenital heart disease by attenuating endoplasmic reticulum stress
}

\author{
YANG ZHOU, WEI-KUN JIA, ZHAO JIAN, LIANG ZHAO, CHEN-CHENG LIU, YONG WANG and YING-BIN XIAO \\ Department of Cardiovascular Surgery, Xinqiao Hospital of The Third Military \\ Medical University, Chongqing 400037, P.R. China
}

Received January 30, 2016; Accepted May 9, 2017

DOI: $10.3892 / \mathrm{mmr} .2017 .6934$

\begin{abstract}
Chronic hypoxia is a key pathological change in patients with cyanotic congenital heart defect (CCHD). It has been demonstrated that enhanced myocardial unfolded protein response (UPR) increases the capacity to buffer endoplasmic reticulum (ER) stress and to avoid subsequent apoptosis caused by the hypoxia that underlies CCHD. The present study was performed to determine the regulatory role of microRNAs (miRNAs) in this cytoprotective UPR process. The results revealed that miR-199a-5p was markedly downregulated in the cardiac tissue of patients with CCHD and in human myocardial cells cultured in hypoxic conditions. The two major UPR modulators, $78 \mathrm{kDa}$ glucose-regulated protein (GRP78) and activating transcription factor 6 (ATF6), were potential target genes of miR-199a-5p in CCHD myocardial specimens. In addition, the miR-199a-5p mimic and inhibitor were evidently able to change GRP78 and ATF6 gene expression and ER stress-associated apoptosis in hypoxia-treated cardiomyocytes. The interaction between miR-199a-5p and the ATF6 and GRP78 3'-UTR binding sites in myocardial cells was also confirmed by luciferase assay. Thus, it is concluded that myocardial downregulation of miR-199a-5p favors the
\end{abstract}

Correspondence to: Professor Ying-Bin Xiao, Department of Cardiovascular Surgery, Xinqiao Hospital of The Third Military Medical University, 183 Xinqiao Street, Chongqing 400037, P.R. China

E-mail: xqxiaoyb@163.com

Abbreviations: ACHD, acyanotic congenital heart defects; ATF6, activating transcription factor 6; CCHD, cyanotic congenital heart defects; ER, endoplasmic reticulum; GRP78, $78 \mathrm{kDa}$ glucose-regulated protein; HCMs, human cardiac myocytes; HIF, hypoxia-inducible factor; miRNAs, microRNAs; $\mathrm{SaO}_{2}$, arterial oxygen saturations; UPR, unfolded protein response; STAT3, signal transducer and activator of transcription 3; UTR, untranslated region

Key words: miR-199a-5p, hypoxia, myocardial protection, unfolded protein response, $78 \mathrm{kDa}$ glucose-regulated protein, activating transcription factor 6
UPR against hypoxia-induced ER stress in CCHD, which contributes to myocardial protection.

\section{Introduction}

Congenital heart defects occur in $\sim 1 \%$ of newborn children and can be divided into two main groups: Cyanotic congenital heart defects (CCHD) and acyanotic congenital heart defects (ACHD) (1). Patients with CCHD often presents with cyanosis that results from systemic hypoxia due to deoxygenated blood bypassing the pulmonary circulation, which is caused by heart structural defects including tetralogy of Fallot (2). The heart of a patient with CCHD is also chronically perfused with hypoxic blood. However, cardiac failure rarely occurs in infants with CCHD, who can survive for a period of time and resist the hypoxic-ischemic challenge caused by cardiac surgery (3). An exploration of the underlying mechanism by which the hearts of children with CCHD adapt to long-term hypoxia may improve cardioprotection in infants with CCHD, but remains to be fully elucidated.

The endoplasmic reticulum (ER), a vital organelle responsible for the correct three-dimensional folding of newly translated proteins into their native conformation, is vulnerable to exogenous toxicants (4) and suboptimal cellular microenvironments, including hypoxia (5). The abnormity of energy metabolism caused by inadequate oxygen can adversely affect ER protein folding and initiate ER stress, and sustained and strong ER stress leads to cell apoptosis. However, cells can initiate the unfolded protein response (UPR) to defuse the crisis of ER stress by reducing the generation of new unfolded proteins, facilitating the refolding of denatured proteins and promoting the degradation of misfolded proteins (6).

A previous study (7) noted the enhanced UPR process in myocardial samples from patients with chronic hypoxic CCHD. The levels of the $78 \mathrm{kDa}$ glucose-regulated protein (GRP78), a major indicator and molecular chaperone of UPR, were higher in cyanotic patients. In addition, among the classic three ER stress sensors, the activating transcription factor 6 (ATF6) pathway was markedly activated in patients with CCHD. In unstressed cells, GRP78 binds to ATF6, which is maintained in an inactive $90 \mathrm{kDa}$ form (ATF6-p90). Upon initial ER stress, the insufficient GRP78 releases ATF6-p90, 
which is translocated from the ER to the Golgi apparatus where it is cleaved into a $50 \mathrm{kDa}$ ATF6-p50 fragment that enters to the nucleus to mediate the transcriptional activation of several genes that ameliorate ER stress, including GRP78 (8). Similarly, another study also reported that enhanced GRP78 and ATF6 protected the heart from ischemic damage (9). However, little is known about the endogenous signals that control GRP78 and ATF6 genes expression, particularly in the hypoxic/ischemic heart.

A number of studies $(10,11)$ suggest the importance of microRNAs (miRNAs) for the regulation of gene expression in cardiac tissues under physiological and pathological conditions, and during the heart development process. A previous study also implicated miRNAs in the regulation of UPR (12). Therefore, the present study sought to identify the miRNAs involved in the regulation of UPR-associated gene expression, including GRP78 and ATF6, within the hypoxic myocardium. miRNA-199a-5p (miR-199a-5p) has been reported to be an abundant miRNA species expressed in myocardium $(13,14)$. The downregulation of miR-199a-5p has been reported to serve an important role in preventing cardiac hypertrophy and subsequent heart failure (15). miR-199a-5p is also involved in pathogenesis of selenium deficiency cardiomyopathy (Keshan disease) (16). Several lines of evidence suggest that miR-199a-5p is sensitive to oxygen tension and that its levels decrease rapidly following hypoxia. Hypoxia-inducible factor- $1 \alpha(\mathrm{HIF}-1 \alpha)$ is a validated target of miR-199a-5p in various cells (17). Furthermore, miR-199a-5p downregulation also results in the upregulation of sirtuin-1, which in turn contributes to HIF-1 $\alpha$ stabilization (18).

Using bioinformatics prediction programs based on the human genome, it was previously identified that oxysensitive miR-199a-5p can simultaneously interact with the 3'-untranslated region (3'-UTR) of GRP78 and ATF6, which has been identified in cell types including prostate cancer cells and monocytes from patients with $\alpha$-1-antitrypsin deficiency $(19,20)$. It is well established that miRNAs are not only expressed differentially, but also act differentially in a tissue- and disease-specific manner $(21,22)$. To the best of our knowledge, there have been no reports on miR-199a-5p-mediated influences on UPR-associated molecules in cardiomyocytes during either normoxia or hypoxia.

The aim of the present study was to determine whether changes in miR-199a-5p serve an important role in regulating UPR in the hearts of patients with CCHD. Initially, the levels of miR-199a-5p and the effect on ATF6 and GRP78 expression in cardiomyocytes from patients with CCHD were evaluated. Subsequently, using an in vitro human cardiomyocyte culture model under normoxic and hypoxic conditions, the biological effects of miR-199a-5p in promoting UPR and apoptosis inhibition by effecting ATF6 and GRP78 expression were demonstrated. The results may demonstrate a novel mechanism for cardioprotection in CCHD.

\section{Materials and methods}

Patients. Subjects with congenital heart disease were recruited from the Department of Cardiovascular Surgery, Xinqiao Hospital of Third Military Medical University
(Chongqing, China) between 2013 and 2014. The two separate cohorts comprised 17 patients with a diagnosis of CCHD and 15 ACHD individuals. Clinical characteristics of the patients are summarized in Table I. The parents of all subjects provided written informed consent and the study protocol was approved by the Ethics Committee of the Third Military Medical University. During cardiac surgery, ventricular specimens were collected from the right ventricular outflow tract immediately following cardiopulmonary bypass. Each specimen was immediately placed in liquid nitrogen for protein and RNA extraction.

Cell culture. Human cardiac myocytes (HCMs; cat. no. C12810) from normal ventricle tissue of the adult heart along with the Myocytes Growth Medium kit (cat. no. C22070) were purchased from PromoCell GmbH (Heidelberg, Germany). The HCMs express markers of cardiac differentiation and possess proliferation capacity. The culture system contained final concentrations of $0.5 \mathrm{ng} / \mathrm{ml}$ epidermal growth factor, $2 \mathrm{ng} / \mathrm{ml}$ basic fibroblast growth factor, $5 \mu \mathrm{g} / \mathrm{ml}$ insulin, $5 \%$ fetal bovine serum (Gibco; Thermo Fisher Scientific, Inc., Waltham, MA, USA) and $0.5 \%$ penicillin/streptomycin. Cells were maintained at $37^{\circ} \mathrm{C}$ under normoxia $\left(21 \% \mathrm{O}_{2}, 5 \% \mathrm{CO}_{2}\right.$ and $\left.74 \% \mathrm{~N}_{2}\right)$, moderate hypoxia $\left(3 \% \mathrm{O}_{2}, 5 \% \mathrm{CO}_{2}\right.$ and $92 \%$ $\mathrm{N}_{2}$ ) or severe hypoxia $\left(1 \% \mathrm{O}_{2}, 5 \% \mathrm{CO}_{2}\right.$ and $\left.94 \% \mathrm{~N}_{2}\right)$. The medium was changed $24 \mathrm{~h}$ after seeding and then every other day. Accutase (Innovative Cell Technologies, Inc., San Diego, CA, USA) was used to detach HCMs from culture plates for subsequent analysis. HEK293T cells (American Type Culture Collection, Manassas VA, USA) used for transfection were cultured in Dulbecco's modified Eagle's medium (Gibco; Thermo Fisher Scientific, Inc.) containing 10\% fetal bovine serum under $5 \% \mathrm{CO}_{2}$ and $37^{\circ} \mathrm{C}$.

RNA extraction and reverse transcription-quantitative polymerase chain reaction $(R T-q P C R)$. Total RNA and small RNA-enriched RNA fractions were extracted using RNAiso (Takara Biotechnology Co., Ltd., Dalian, China) and the PureLink miRNA Isolating kit (Invitrogen; Thermo Fisher Scientific, Inc.), respectively. RNA samples were reverse transcribed using the PrimeScript ${ }^{\circledR}$ RT reagent kit (Takara Biotechnology Co., Ltd.). RT-qPCR was performed to analyze ATF6 and GRP78 mRNA expression using the Power SYBR Green PCR Master Mix kit (Applied Biosystems; Thermo Fisher Scientific, Inc.). The following PCR primers were used in the experiments: i) GRP78, forward 5'-GATAATCAACCAACTGTTAC-3' and reverse 5'-GTA TCCTCTTCACCAGTTGG-3'; ii) ATF6, forward 5'-TGAACT TCGAGGATGGGTTC-3' and reverse 5'-TCACTCCCTGAG TTCCTGCT-3' and iii) GAPDH, forward 5'-CGGATTTGG TCGTATTGGG-3' and reverse 5'-TCTCGCTCCTGGAAG ATGG-3'. Mature miR-199a-5p levels were measured using the GeneCopoeia All-in-One miRNA qRT-PCR Detection kit (GeneCopoeia, Inc., Rockville, MD, USA). GAPDH mRNA and U6 snRNA were used as loading controls. Relative expression analysis was performed using the $2^{-\Delta \Delta \mathrm{Cq}}$ method (23).

Transfection of miR-199a-5p mimic and inhibitor. The miR-199a-5p mimic, miR-199a-5p inhibitor and negative controls were obtained from Shanghai GenePharma Co., 
Table I. Clinical characteristics of patients.

\begin{tabular}{|c|c|c|}
\hline Characteristic & Acyanotic $(n=15)$ & Cyanotic $(n=17)$ \\
\hline Age at surgery (year) ${ }^{\mathrm{a}}$ & $6.7(2.6-17)$ & $7.5(2.9-16)$ \\
\hline Weight at surgery $(\mathrm{kg})^{\mathrm{a}}$ & $17.6(12.8-52.8)$ & $15.7(11.4-49.2)$ \\
\hline Artery oxygen saturation ${ }^{\mathrm{b}}$ & $97.3 \pm 1.3$ & $84.4 \pm 5.5^{\mathrm{d}}$ \\
\hline Hemoglobin $(\mathrm{g} / \mathrm{dl})^{\mathrm{b}}$ & $12.6 \pm 1.1$ & $16.9 \pm 1.8^{\mathrm{d}}$ \\
\hline \multicolumn{3}{|l|}{ Diagnoses $^{c}$} \\
\hline Ventricular septal defect & 6 & 0 \\
\hline Atrial septal defect & 4 & 0 \\
\hline Tetralogy of Fallot & 0 & 17 \\
\hline Pulmonary atresia with ventricular septal defect & 2 & 0 \\
\hline Pulmonary arteriovenous malformation & 3 & 0 \\
\hline
\end{tabular}

Data are given as ${ }^{\mathrm{a}}$ medians with ranges, ${ }^{\mathrm{b}}$ mean \pm standard deviation or ${ }^{\mathrm{c}}$ number of patients. ${ }^{\mathrm{d}} \mathrm{P}<0.01 \mathrm{vs}$. acyanotic patients.

Ltd. (Shanghai, China). The sequences were as follows: Has-miR-199a-5p mimic, 5'-CCCAGUGUUCAGACUACC UGUUC-3' (Sense), 5'-ACAGGUAGUCUGAACACUGGG UU-3' (Anti-sense); mimic negative control, 5'-UUCUCCGAA CGUGUCACGUTT-3' (Sense), 5'-ACGUGACACGUUCGG AGAATT-3' (Anti-sense); Has-miR-199a-5p inhibitor, 5'-GAA CAGGUAGUCUGAACACUGGG-3'; and inhibitor negative control, 5'-CAGUACUUUUGUGUAGUACAA-3'. HCM cells and HEK293 cells were transfected $24 \mathrm{~h}$ after seeding into 6 -well plates at a density of $1.2 \times 10^{5}$ cells/well. Transfection of miR-199a-5p mimic (100 nM) and inhibitor (100 nM) with a negative control were performed using Lipofectamine 2000 (Invitrogen; Thermo Fisher Scientific, Inc.). After 6 h, the transfection solution was removed and replaced with $2 \mathrm{ml}$ fresh medium. Transfection efficiency $(>90 \%)$ was validated by RT-qPCR prior to proceeding to further experiments (data not shown).

Western blot analysis. Rabbit polyclonal antibodies against GRP78 and GAPDH (cat. nos. ab108613 and ab9485; Abcam, Cambridge, MA, USA), C/EBP homologous protein (CHOP), ATF6-p90 and ATF6-p50 (cat. nos. sc793 and sc14253; Santa Cruz Biotechnology, Dallas, TX, USA) were used as primary antibodies. Total protein was extracted using a radioimmunoprecipitation assay lysis buffer (cat. no. P0013B; Beyotime Institute of Biotechnology, Haimen, China), and a bicinchoninic acid concentration measurement kit (cat. no. P0012; Beyotime Institute of Biotechnology) was used for the protein determination. A 5\% gel for concentration and an $8 \%$ gel for separation were selected for SDS-PAGE, and 50-70 $\mu \mathrm{g}$ total protein was loaded per lane. For blotting, the proteins were transferred to polyvinylidene difluoride membranes by wet transfer. The free sites were blocked with 5\% non-fat milk powder for $1 \mathrm{~h}$ at room temperature, and the membrane was incubated in primary antibody diluted $(1: 1,000)$ in Primary Antibody Dilution Buffer (cat. no. P0023A; Beyotime Institute of Biotechnology) at $4^{\circ} \mathrm{C}$ overnight. Following washing 3 times for $10 \mathrm{~min}$ in TBS with Tween, the membranes were incubated with a horseradish peroxidase-conjugated goat anti-rabbit immunoglobulin G secondary antibody (cat. no. A0208; diluted 1:1,000; Beyotime Institute of Biotechnology) for $1 \mathrm{~h}$ at $37^{\circ} \mathrm{C}$. Protein bands were visualized using a Pierce Fast Western Blot kit, ECL Substrate (cat. no. 35055; Thermo Fisher Scientific, Inc.). The intensity of the individual bands was measured with a ChemiDoc XRS system using the Quantity One (version 4.6.3; Bio-Rad Laboratories, Inc., Hercules, CA, USA) or ImageJ (version 1.45s; National Institutes of Health, Bethesda, MD, USA) software.

Apoptosis assay. An Annexin V-FITC/propidium iodide (PI) apoptosis detection kit (BD Biosciences Pharmingen, San Diego, CA, USA) was used according to the manufacturer's protocol. Immunofluorescent staining of cells was analyzed by flow cytometry (Beckman Coulter, Brea, CA, USA).

Luciferase reporter plasmid transfection. The 3'-UTR of ATF6 or GRP78 containing a miR-199a-5p-binding site amplified from HCM cell cDNA or the mutant UTRs with 6-bp deletions in the binding site (positions 2-7 of the seed region) was cloned into the Xba1 site of pGL3-promoter vector (Promega Corporation, Madison, WI, USA). The primers were used for the cloning were reported previously by Dai et al (24): GRP78 3'UTR, forward 5'-TCTAGACTTTTCATTAGCAGT TGCTCACA-3' and reverse 5'-TCTAGACCCAACATACCA AATACTCCCTC-3'; ATF6 3'UTR, forward 5'-TCTAGAGAT CAATGGGCAGGACTACGA-3' and reverse 5'-TCTAGA CCAAATAGATGGGTAGATGATGAAA-3'. The deletion mutations of the 3'-UTR were introduced by the design of suitable PCR primers. The PCR experiments and construction of the 3'UTR-containing plasmid based on pmiR-RB-Report ${ }^{\mathrm{TM}}$ were completed by Guangzhou RiboBio Co., Ltd. (Guangzhou, China). The constructed vectors were co-transfected with miR-199a-5p mimics into human embryonic kidney (HEK) 293T cells or HCM cells using Lipofectamine 2000 (Invitrogen; Thermo Fisher Scientific, Inc.). Empty vector and pRL-TK (Promega Corporation) were added as the negative and internal controls. Cells were lysed after $48 \mathrm{~h}$ of transfection and the activities of the firefly and Renilla luciferases were measured consecutively using the Dual-Luciferase Reporter Assay System (Promega Corporation). 
A

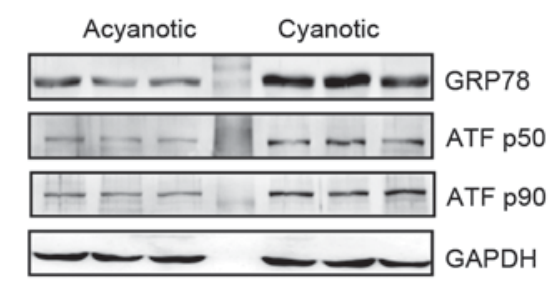

B

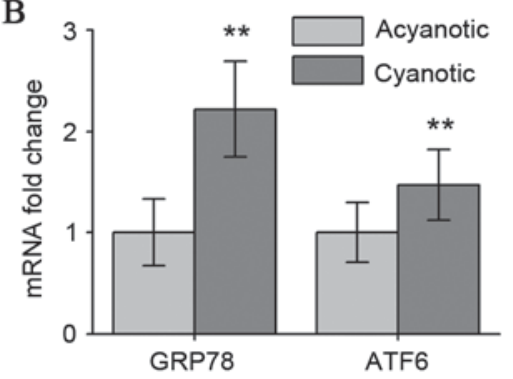

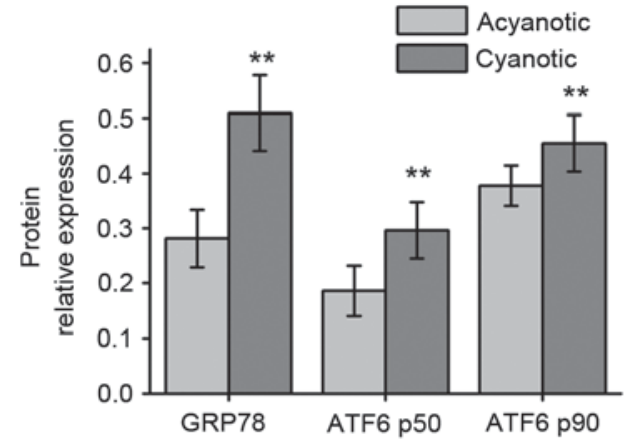

C

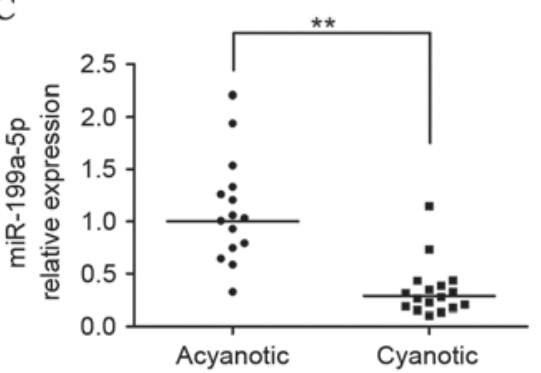

$\mathrm{D}$

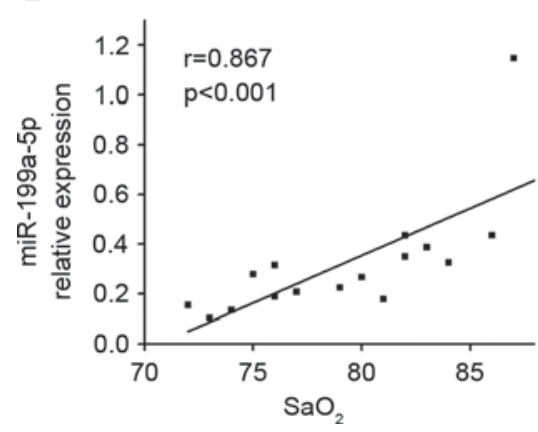

$\mathrm{E}$

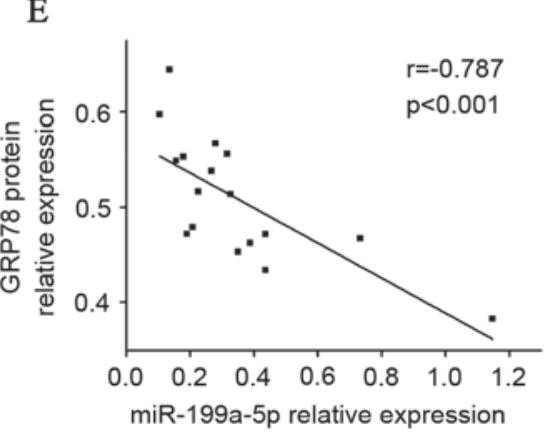

F

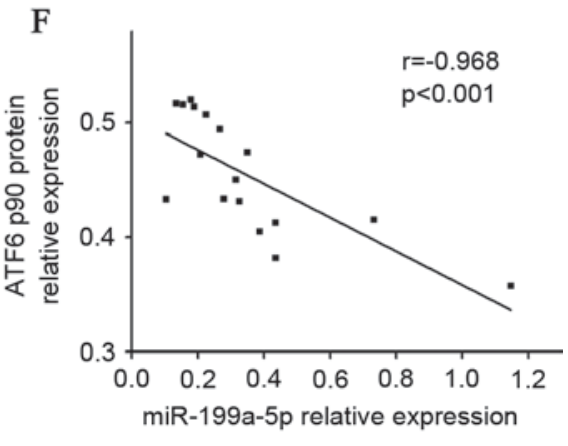

Figure 1. Myocardial ATF6 and GRP78 expression and miR-199a-5p levels in patients with cyanotic CHD and a cyanotic CHD. (A) Representative western blot analysis and the group data of GRP78 and ATF6 (including full-length p90 and cleaved p50) protein expression in patients with cyanotic CHD and a cyanotic CHD. (B) RT-qPCR revealed differences in cardiac ATF6 and GRP78 mRNA expression between the two groups. (C) Individual data derived from the RT-qPCR analysis for miR-199a-5p expression in the myocardium from patients of the two groups. (D) Correlation between myocardial miR-199a-5p expression levels and $\mathrm{SaO}_{2}$ was observed in patients with cyanotic CHD. miR-199a-5p negatively correlated with (E) GRP78 and (F) ATF6 $\alpha$-p90 protein levels in the heart tissue of patients with cyanotic CHD. Data are presented as the mean \pm standard deviation ( $\mathrm{n}=17$ for CCHD and $\mathrm{n}=15$ for ACHD). Spearman's correlation coefficient and $\mathrm{P}$-values for each correlation analysis are presented. ${ }^{* *} \mathrm{P}<0.01$ vs. the ACHD group. ATF6, activating transcription factor 6; GRP78, $78 \mathrm{kDa}$ glucose-regulated protein; miR, microRNA; CHD, congenital heart defects; RT-qPCR, reverse transcription-quantitative polymerase chain reaction.

Statistical analysis. Results are presented as the mean \pm standard deviation. Differences were evaluated by the unpaired Student's t-test for comparing the means of the two groups or one-way analysis of variance followed by a least significance difference test for multiple comparisons. Statistical analyses were performed with SPSS software version 13.0 (SPSS, Inc., Chicago, IL, USA). P<0.05 was considered to indicate a statistically significant difference.

\section{Results}

Expression of miR-199a-5p and its potential targets differ in CCHD and ACHD myocardial tissues. Western blot analysis indicated that the CCHD cardiac specimens exhibited higher GRP78 protein levels compared with the ACHD group. Adaptation to chronic hypoxia in CCHD hearts also resulted in higher p90 full-length ATF6 (native translational form) and p50 cleaved ATF6 (active form) protein levels (Fig. 1A). RT-qPCR results indicated that the CCHD group exhibited a significant increase in GRP78 and ATF6 mRNA levels compared with the ACHD group (Fig. 1B). A 5-fold downregulation in miR-199a-5p levels in CCHD patients was also observed (Fig. 1C). Among the CCHD group, myocardial miR-199a-5p expression correlated well with arterial oxygen saturation $\left(\mathrm{SaO}_{2}\right)$, indicating that the degree of hypoxia was associated with decreased miR-199a-5p (Fig. 1D). When exploring whether miR-199a-5p may be clinically relevant to GRP78 and ATF6 expression, an inverse correlation between miR-199a-5p levels and these two protein levels in CCHD patients was identified (Fig. 1E and F).

Moderate hypoxia enhances anti-apoptotic UPR and downregulates miR-199a-5p in cultured human cardiomyocytes. Subsequently, suitable in vitro models were established to simulate CCHD by culturing HCM cells in different oxygen environments $\left(21,3\right.$ and $\left.1 \% \mathrm{O}_{2}\right)$ for 5 days. The results demonstrated that Annexin $\mathrm{V}^{+}$apoptotic rates markedly increased under $1 \% \mathrm{O}_{2}$ hypoxia compared with $21 \% \mathrm{O}_{2}$ normoxia; however, the 


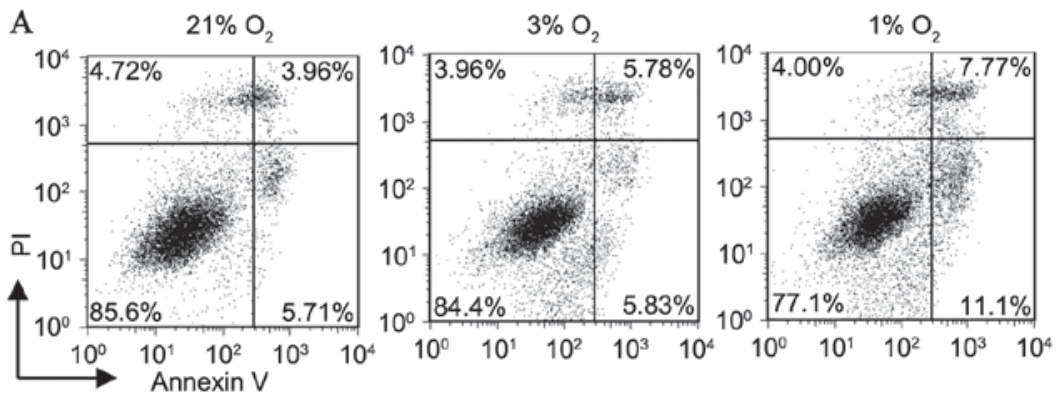

B
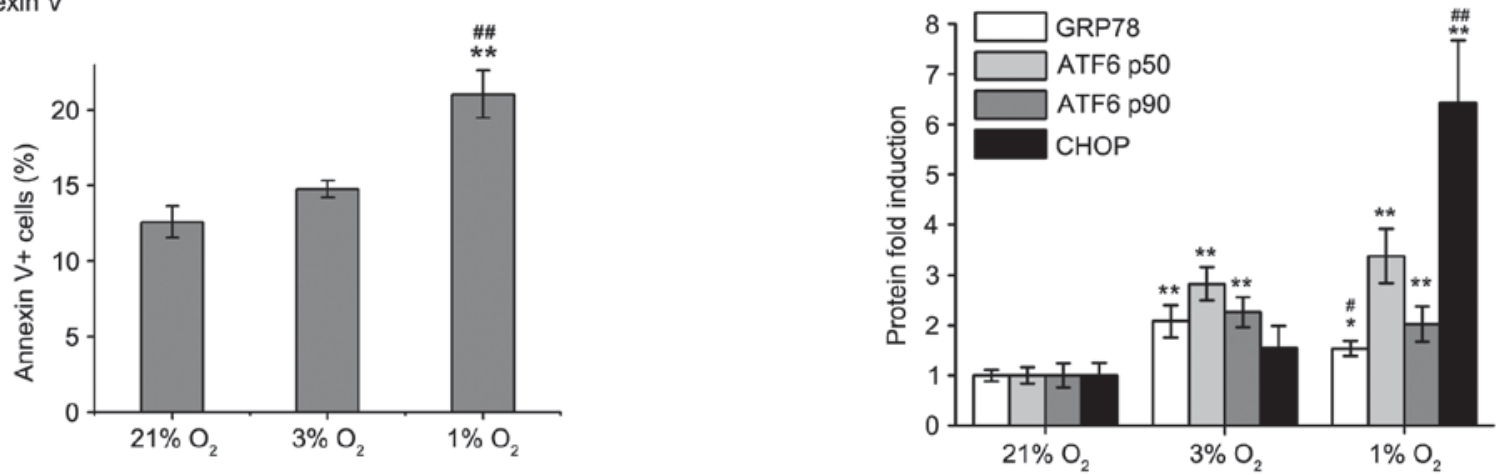

$\mathrm{C}$

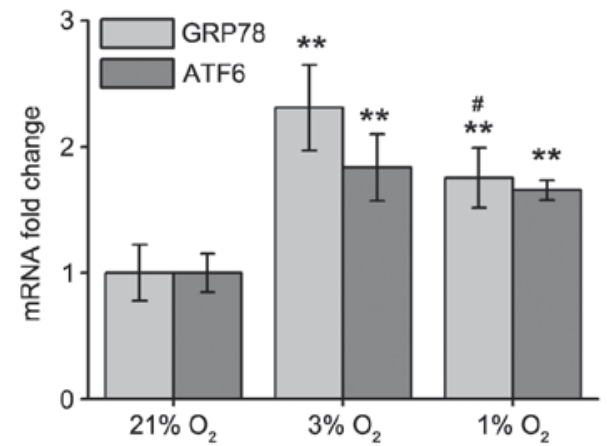

D

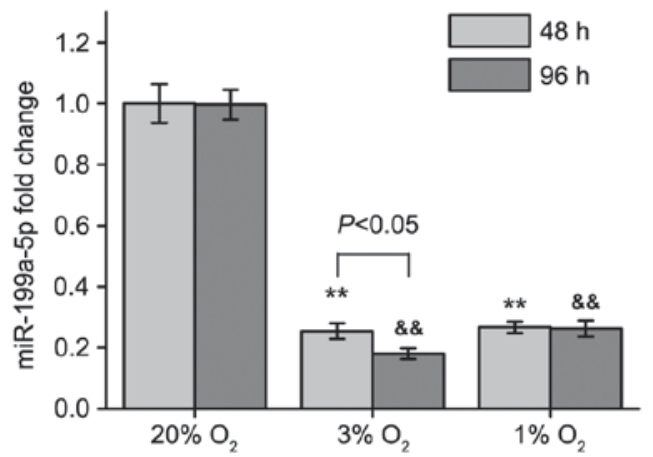

Figure 2. Moderate and severe hypoxia cause different apoptosis rates and changes in GRP78, ATF6 and miR-199a-5p levels. (A) Flow cytometry plots of Annexin V/PI staining in $\mathrm{HCM}$ cells following 21, 3 and $1 \% \mathrm{O}_{2}$ treatments for 5 days. Percentages of all Annexin $\mathrm{V}^{+}\left(\mathrm{PI}^{+/}\right)$apoptotic cells were evaluated (B) Western blot analysis of GRP78, ATF6 $\alpha$-p50, ATF6 $\alpha$-p90 and CHOP protein expression in HCM cells cultured under different oxygen tension. (C) Induction of GRP78 and ATF6 mRNA expression in HCM cells following varying degrees of hypoxia as detected by reverse transcription-quantitative polymerase chain reaction. (D) miR-199a-5p expression levels were determined in 48 and $96 \mathrm{~h}$ hypoxia-cultured HCM cells. Values presented as the mean \pm standard deviation from three independent experiments. ${ }^{*} \mathrm{P}<0.05,{ }^{* *} \mathrm{P}<0.01$ and ${ }^{\& \&} \mathrm{P}<0.01$ compared with the respective $21 \% \mathrm{O}_{2}$-cultured group; ${ }^{\#} \mathrm{P}<0.05$ and ${ }^{\# \#} \mathrm{P}<0.01$ vs. the $3 \%$ $\mathrm{O}_{2}$-cultured group. HCM, human cardiac myocyte; PI, propidium iodide; GRP78, 78 kDa glucose-regulated protein; ATF6, activating transcription factor 6; CHOP, C/EBP homologous protein; miR, microRNA.

apoptotic rate did not significantly increase at $3 \% \mathrm{O}_{2}$ compared with $21 \% \mathrm{O}_{2}$ (Fig. 2A). Additionally, the protein expression of CHOP, a pro-apoptotic transcription factor induced by ER stress, increased significantly following culture in $1 \% \mathrm{O}_{2}$ conditions, but was not significantly altered by $3 \% \mathrm{O}_{2}$ compared with normoxic conditions (Fig. 2B). Notably, GRP78 expression at the mRNA and protein levels was significantly higher under $3 \% \mathrm{O}_{2}$ compared with $1 \% \mathrm{O}_{2}$ culture. ATF6 expression was increased by hypoxia ( 3 and $1 \%$ ) compared with $21 \% \mathrm{O}_{2}$, and not different between the 3 and $1 \% \mathrm{O}_{2}$ groups (Fig. 2B and $\mathrm{C}$ ). This suggested that $3 \% \mathrm{O}_{2}$ may invoke the protective roles of UPR under hypoxia, while $1 \% \mathrm{O}_{2}$ resulted in decompensated ER stress and cell apoptosis. Thus, it was hypothesized that moderate in vitro hypoxia $\left(3 \% \mathrm{O}_{2}\right)$ represented the pathological process observed in patients with CCHD.

As demonstrated in Fig. 2D, exposure to low oxygen levels decreased miR-199a-5p levels. Culture in $1 \% \mathrm{O}_{2}$ did not have a greater effect on miR-199a-5p levels compared with culture in $3 \% \mathrm{O}_{2}$. Additionally, for the $3 \% \mathrm{O}_{2}$ group, prolonged $(96 \mathrm{~h})$ hypoxia was more potent at inhibiting miR-199a-5p than short-term (48 h) hypoxia. These results indicate that moderate and persistent hypoxia in patients with CCHD is enough to maintain miR-199a-5p at a significantly reduced level.

miR-199a-5p overexpression decreased hypoxia-induced ATF6 and GRP78 expression and increased apoptosis. To explore whether hypoxia-induced ATF6 and GRP78 expression can be modulated by miR-199a-5p, exogenously synthetic miRNA mimic and inhibitor were transfected to enhance or knockdown miR-199a-5p levels in HCM cells cultured under $3 \% \mathrm{O}_{2}$ for $96 \mathrm{~h}$. As presented in Fig. 3A and B, it was identified that transfection with the miR-199a-5p mimic significantly reduced hypoxic induction of ATF6 and GRP78 protein and mRNA expression compared with the negative mimic control. 
A

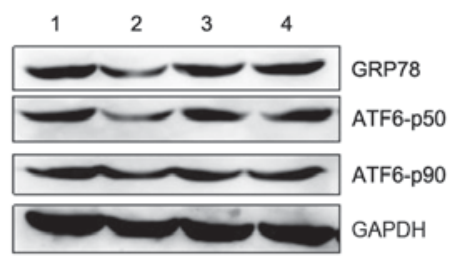

$\mathrm{C}$
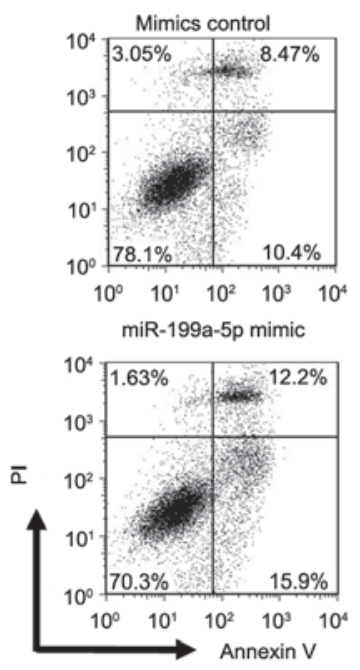

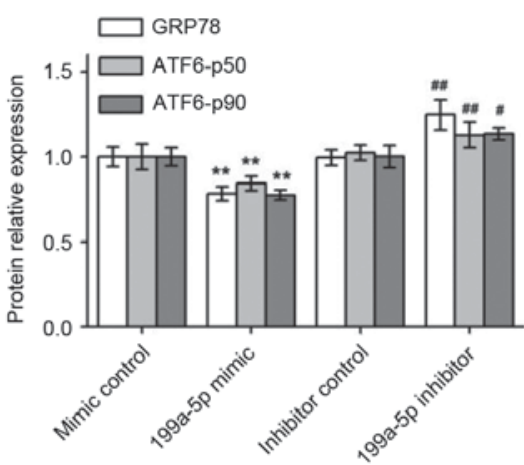

D
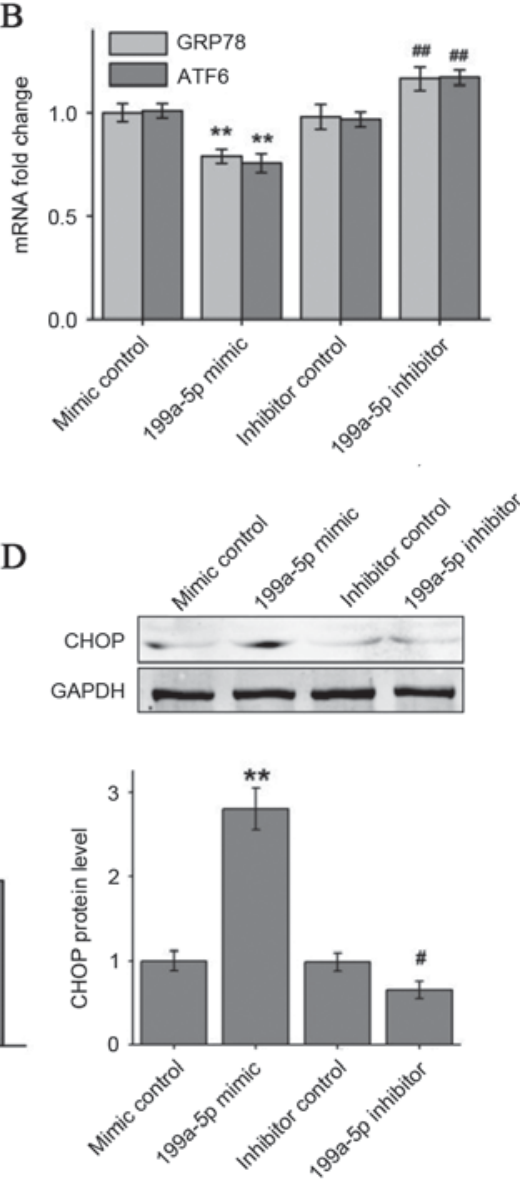

Figure 3. miR-199a-5p mimic and inhibitor affect ER stress-associated apoptosis by regulating the expression of GRP78 and ATF6. (A) GRP78 and full-length and cleaved ATF6 protein levels and (B) GRP78 and ATF6 mRNA levels were measured by western blotting and reverse transcription-quantitative polymerase chain reaction, respectively, in 3\% $\mathrm{O}_{2}$ cultured HCM cells transfected with miR-199a-5p mimic or miR-199a-5p inhibitor. Numbers 1-4 at the top of the western blot lanes indicate the mimic control, miR-199a-5p mimic, the inhibitor control and the miR-199a-5p inhibitor groups, respectively. (C) miR-199a-5p mimic significantly increased hypoxia-induced cell apoptosis and miR-199a-5p inhibitor reduced apoptosis in hypoxic cells. (D) miR-199a-5p mimic or inhibitor changed $\mathrm{CHOP}$ expression in $3 \% \mathrm{O}_{2}$ cultured $\mathrm{HCM}$ cells. Data are presented as the mean \pm standard deviation of three independent experiments. ${ }^{* *} \mathrm{P}<0.01$ vs. mimic control group; ${ }^{*} \mathrm{P}<0.05$ and ${ }^{\# / 2} \mathrm{P}<0.01$ vs. inhibitor control group. miR, microRNA; ER, endoplasmic reticulum; HCMs, human cardiac myocyte; GRP78, $78 \mathrm{kDa}$ glucose-regulated protein; ATF6, activating transcription factor 6; PI, propidium iodide; CHOP, C/EBP homologous protein.

By contrast, the miR-199a-5p inhibitor, but not the inhibitor control, increased ATF6 and GRP78 expression in hypoxic HCMs. Flow cytometry analysis indicated an increased percentage of Annexin $\mathrm{V}^{+}$apoptotic HCMs under hypoxia when overexpressing miR-199a-5p. By contrast, HCMs transfected with the miR-199a-5p inhibitor demonstrated a significant reduction in apoptosis percentages under hypoxia compared with the inhibitor control (Fig. 3C). Pro-apoptotic CHOP protein levels also exhibited varying trends similar to the flow cytometry results following transfection with miR-199a-5p mimic and inhibitor in hypoxia-cultured HCM cells (Fig. 3D).

GRP78 and ATF6 were confirmed to be target genes for miR-199a-5p. To confirm whether miR-199a-5pcan directly targetthe ATF6 and GRP78 3'UTRs, a luciferase reporter plasmid containing wild type ATF6 or GRP78 3'-UTR, and mutants lacking the miR-199a-5p binding sites were co-transfected with the miR-199a-5p mimic into HEK293T and HCM cells. It was first observed that the miR-199a-5p mimic downregulated the relative luciferase activity of wild-type GRP78 and ATF6 in HEK293T cells cultured in normoxia, a well-established and widely used protocol for miRNA luciferase reporter analysis. By contrast, co-transfection of miR-199a-5p mimics and the vector lacking any 3'-UTR sequences (mutant control) demonstrated no such repressive effects (Fig. 4A). The results in the human myocardial cell line also demonstrated that miR-199a-5p significantly decreased luciferase gene expression in HCM cells transfected with the reporter vectors containing the ATF6 or GRP78 3'-UTRs compared with the mutant controls and that the inhibition effect was more pronounced compared with the HEK293T cells (Fig. 4B). The effects of endogenous miR-199a-5p on the GRP78 and ATF6 3'-UTR reporters in HCM cells under normoxia and hypoxia were also examined. In HCM cells under $3 \% \mathrm{O}_{2}$ hypoxia and following reporter transfection, the wild-type reporters were less repressed compared with the $21 \% \mathrm{O}_{2}$ group (Fig. $4 \mathrm{C}$ ). These results demonstrated that exogenous miR-199a-5p can suppress GRP78 and ATF6 expression in normoxic myocardial cells by targeting their 3'-UTRs, and 

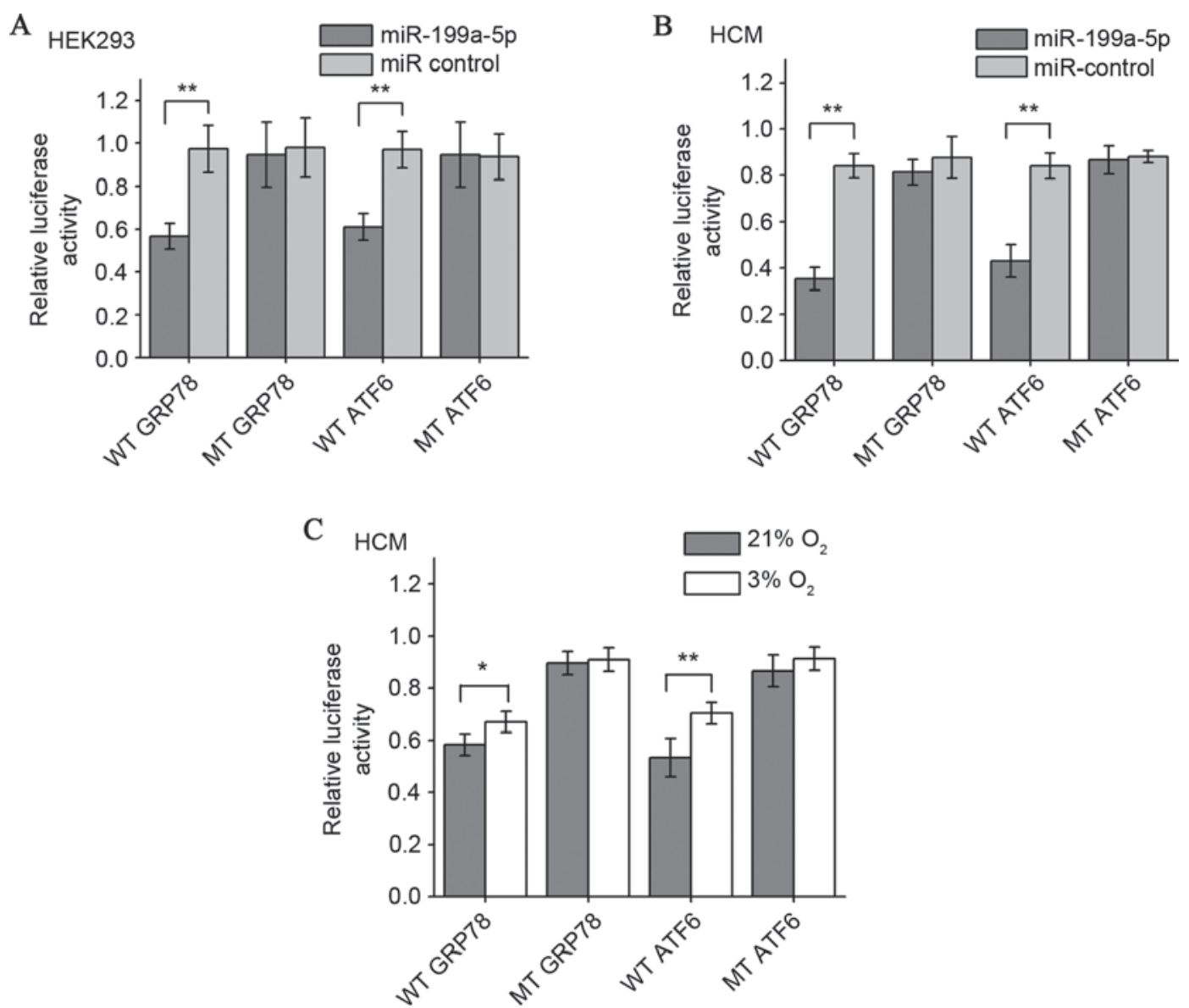

Figure 4. Establishment of GRP78 and ATF6 as target genes for miR-199a-5p by luciferase reporter gene assay. miR-199a-5p mimics decreased the expression of luciferase activities elicited by vectors containing the 3'-UTR of wild-type GRP78 and ATF6 mRNAs, but not 3'UTR binding site mutants in (A) HEK293 cells and (B) HCM cells. Relative luciferase activity was determined by normalizing firefly to Renilla (control) luciferase activity. (C) HCM cells transfected with a reporter containing either wild-type or mutant GRP78 or ATF6 3'-UTRs were cultured under 21 or $3 \% \mathrm{O}_{2}$ for $48 \mathrm{~h}$. The effect of different endogenous miR-199a-5p levels under normoxia and hypoxia on luciferase activity was compared. ${ }^{*} \mathrm{P}<0.05$ and ${ }^{* *} \mathrm{P}<0.01$. Data are presented as the mean \pm standard deviation of three independent experiments. 3'-UTR, 3'-untranslated region; WT, wild-type 3'-UTR; GRP78, 78 kDa glucose-regulated protein; MT, mutant 3'-UTR; ATF6, activating transcription factor 6; miR, microRNA; UTR, untranslated region; HEK, human embryonic kidney cells; HCM, human cardiac myocyte.

that hypoxia weakens this suppressive effect by downregulating intracellular miR-199a-5p levels.

\section{Discussion}

To the best of our knowledge, the present study is the first to report a mechanism whereby oxygen-sensitive miR-199a-5p levels change and regulate UPR activation by controlling GRP78 and ATF6, the two major ER stress sensors and UPR effectors, thus protecting CCHD patient cardiac myocytes during chronic hypoxia.

A previous study (25) confirmed that the ATF6 pathway was activated in the hypoxic myocardium of patients with CCHD. In addition to functional ATF6-p50, enhanced gene expression of native ATF6-p90 was also useful in promoting the persistent UPR process by generating ATF6-p50. It was noted that ATF6 p90 and p50 were consistently changed in the cardiac tissue of patients with CCHD and in hypoxic cultured myocardial cells. In addition, the level of GRP78, a universal ER stress sensor and cytoprotective ER chaperone closely associated with, but not limited to, the ATF6 pathway, was also observed to be upregulated in CCHD. It remains to be elucidated how GRP78 and ATF6 expression are induced by hypoxia, although HIFs are key modulators for many genes during low oxygen conditions. It has been reported (26) that there are no HIF binding sites in the GRP78 promoter and extrinsic HIFs have no effect on the GRP78 promoter activity. Additionally, until now there have been no reports that ATF6 expression is directly regulated by HIFs. Thus, it is possible that GRP78 and ATF6 expression under hypoxic stress is mainly regulated in a HIF-independent manner.

miRNAs fine-tune the activity of various protein-coding genes. A recent study (27) reported that miR-199a-5p is sensitive to hypoxia and decreases quickly to very low levels, resulting in the release of mRNA targets from its inhibitory effect, which has already been demonstrated to be an important mechanism for the promotion of hypoxia-induced gene expression, as in the case of HIF-1 $\alpha$ and vascular endothelial growth factor. The present study demonstrated that cardiac tissue expression of miR-199a-5p was markedly decreased in CCHD individuals undergoing chronic hypoxia. There is a strong linear association between miR-199a-5p levels and the expression of its potential targets GRP78 and ATF6, in addition to the oxygen saturation that may clinically distinguish patients with CCHD from other congenital heart defects. Notably, to the best of our knowledge, the present 
study has obtained the first evidence to demonstrate the causal association between decreased miR-199a-5p levels, and upregulated GRP78 and ATF6 expression by conducting experiments in hypoxic cultured HCMs and by luciferase reporter gene assays. It is possible that cardiac-enriched miR-199a-5p imposes an important repression of GRP78 and ATF6 to basal expression under normoxic conditions. In hypoxia, the downregulation of miR-199a-5p partially represses this effect, resulting in an increase in expression of the two genes.

Although it has been reported (22) that miR-199a-5p can affect the expression of GRP78 and ATF6 in a number of cell types, the present study confirmed this regulation mechanism for the first time in cardiomyocytes. The different luciferase reporter results between HEK293 and HCM cells suggest that these regulatory roles may be more pronounced in myocardial cells compared with other cells. The reason may be that the particular cellular contexts in cardiomyocytes are particularly propitious for miR-199a-5p to target the GRP78 and ATF6 3'-UTRs. miRNAs post-transcriptionally downregulate gene expression by either degrading or translationally repressing target mRNAs. This is dependent upon the complementarity, number of base pairs and accessibility of the binding sites. As the mRNA and protein variation of GRP78 and ATF6 (p90) in the present study exhibited a largely synchronous trend in vivo and in vitro, particularly when exogenously altering the miR-199a-5p level, it was suggested that translational repression, in addition to mRNA destabilization, may be involved in the regulatory mechanism of GRP78 and ATF6 expression by miR-199a-5p (28).

The results of the present study also demonstrated that chronic and moderate hypoxia, as observed in clinical patient with CCHD, can persistently maintain myocardial miR-199a-5p at lower levels. Low oxygen availability induces changes (up- or downregulation) in the expression of a specific subset of miRNAs known as hypoxia miRs (29). Transcription factors, including HIFs, can directly activate the transcription of a subset of upregulated hypoxia miRs including miR-210. However, the mechanisms by which hypoxia selectively represses other hypoxia miRs have been less well-characterized. Haghikia et al (30) demonstrated that signal transducer and activator of transcription 3 (STAT3) protein acts as a potent suppressor of miR-199a-5p transcription. Coincidentally, the activation of the STAT3 signal pathway in patients with CCHD has been previously identified (31). It is speculated that miR-199a-5p is negatively regulated by an enhanced STAT3 pathway under hypoxic conditions, although this requires further study for verification.

In summary, the results of the present study provide a novel adaptation mechanism of cardiac myocytes to chronic hypoxia in patients with CCHD. It is proposed that miR-199a-5p may be a new potential clinical diagnostic and therapeutic target for the protection of cardiocytes against hypoxia.

\section{Acknowledgements}

The present study was supported the National Natural Science Foundation of China (grant no. 81270228).

\section{References}

1. van der Bom T, Zomer AC, Zwinderman AH, Meijboom FJ, Bouma BJ and Mulder BJ: The changing epidemiology of congenital heart disease. Nat Rev Cardiol 8: 50-60, 2011.

2. Miyague NI, Cardoso SM, Meyer F, Ultramari FT, Araújo FH, Rozkowisk I and Toschi AP: Epidemiological study of congenital heart defects in children and adolescents. Analysis of 4,538 cases. Arq Bras Cardiol 80: 269-278, 2003.

3. Oechslin E: Management of adults with cyanotic congenital heart disease. Heart 101: 485-494, 2015.

4. Lou Y, Wang Z, Xu Y, Zhou P, Cao J, Li Y, Chen Y, Sun J and Fu L: Resveratrol prevents doxorubicin-induced cardiotoxicity in $\mathrm{H} 9 \mathrm{c} 2$ cells through the inhibition of endoplasmic reticulum stress and the activation of the Sirtl pathway. Int J Mol Med 36: 873-880, 2015.

5. Schönenberger MJ and Kovacs WJ: Hypoxia signaling pathways: Modulators of oxygen-related organelles. Front Cell Dev Biol 3: 42, 2015.

6. Fu XL and Gao DS: Endoplasmic reticulum proteins quality control and the unfolded protein response: The regulative mechanism of organisms against stress injuries. Biofactors 40: 569-585, 2014.

7. Jian Z, Li JB, Ma RY, Chen L, Wang XF and Xiao YB: Pivotal role of activating transcription factor $6 \alpha$ in myocardial adaptation to chronic hypoxia. Int J Biochem Cell Biol 44: 972-979, 2012.

8. Glembotski CC: Roles for ATF6 and the sarco/endoplasmic reticulum protein quality control system in the heart. J Mol Cell Cardiol 71: 11-15, 2014.

9. Thuerauf DJ, Marcinko M, Gude N, Rubio M, Sussman MA and Glembotski CC: Activation of the unfolded protein response in infarcted mouse heart and hypoxic cultured cardiac myocytes. Circ Res 99: 275-282, 2006.

10. Zhou J, Dong X, Zhou Q, Wang H, Qian Y, Tian W, Ma D and Li X: microRNA expression profiling of heart tissue during fetal development. Int J Mol Med 33: 1250-1260, 2014.

11. Azzouzi HE, Leptidis S, Doevendans PA and De Windt LJ: HypoxamiRs: Regulators of cardiac hypoxia and energy metabolism. Trends Endocrinol Metab 26: 502-508, 2015.

12. Maurel $M$ and Chevet E: Endoplasmic reticulum stress signaling: The microRNA connection. Am J Physiol Cell Physiol 304: C1117-C1126, 2013.

13. Topkara VK and Mann DL: Role of microRNAs in cardiac remodeling and heart failure. Cardiovasc Drugs Ther 25: 171-182, 2011.

14. Lee DS, Chen JH, Lundy DJ, Liu CH, Hwang SM, Pabon L, Shieh RC, Chen CC, Wu SN, Yan YT, et al: Defined MicroRNAs induce aspects of maturation in mouse and human embryonic-stem-cell-derived cardiomyocytes. Cell Rep 12: 1960-1967, 2015.

15. Song XW, Li Q, Lin L, Wang XC, Li DF, Wang GK, Ren AJ, Wang YR, Qin YW, Yuan WJ and Jing Q: MicroRNAs are dynamically regulated in hypertrophic hearts, and miR-199a is essential for the maintenance of cell size in cardiomyocytes. J Cell Physiol 225: 437-443, 2010.

16. Xing Y, Liu Z, Yang G, Gao D and Niu X: MicroRNA expression profiles in rats with selenium deficiency and the possible role of the Wnt/ $\beta$-catenin signaling pathway in cardiac dysfunction. Int J Mol Med 35: 143-152, 2015.

17. Ding G, Huang G, Liu HD, Liang HX, Ni YF, Ding ZH, Ni GY and Hua HW: MiR-199a suppresses the hypoxia-induced proliferation of non-small cell lung cancer cells through targeting HIF1 $\alpha$. Mol Cell Biochem 384: 173-180, 2013.

18. Rane S, He M, Sayed D, Vashistha H, Malhotra A, Sadoshima J, Vatner DE, Vatner SF and Abdellatif M: Downregulation of miR-199a derepresses hypoxia-inducible factor-1alpha and Sirtuin 1 and recapitulates hypoxia preconditioning in cardiac myocytes. Circ Res 104: 879-886, 2009.

19. Su SF, Chang YW, Andreu-Vieyra C, Fang JY, Yang Z, Han B, Lee AS and Liang G: miR-30d, miR-181a and miR-199a-5p cooperatively suppress the endoplasmic reticulum chaperone and signaling regulator GRP78 in cancer. Oncogene 32: 4694-4701, 2013.

20. Hassan T, Carroll TP, Buckley PG, Cummins R, O'Neill SJ, McElvaney NG and Greene CM: miR-199a-5p silencing regulates the unfolded protein response in chronic obstructive pulmonary disease and $\alpha 1$-antitrypsin deficiency. Am J Respir Crit Care Med 189: 263-273, 2014. 
21. Liang Y, Ridzon D, Wong L and Chen C: Characterization of microRNA expression profiles in normal human tissues. BMC Genomics 8: 166, 2007.

22. Nam JW, Rissland OS, Koppstein D, Abreu-Goodger C, Jan CH, Agarwal V, Yildirim MA, Rodriguez A and Bartel DP: Global analyses of the effect of different cellular contexts on microRNA targeting. Mol Cell 53: 1031-1043, 2014.

23. Livak KJ and Schmittgen TD: Analysis of relative gene expression data using real-time quantitative PCR and the 2(-Delta Delta C(T)) Method. Methods 25: 402-408, 2001.

24. Dai BH, Geng L, Wang Y, Sui CJ, Xie F, Shen RX, Shen WF and Yang JM: microRNA-199a-5p protects hepatocytes from bile acid-induced sustained endoplasmic reticulum stress. Cell Death Dis 4: e604, 2013

25. Namba T, Ishihara $T$, Tanaka $K$, Hoshino $T$ and Mizushima $T$ : Transcriptional activation of ATF6 by endoplasmic reticulum stressors. Biochem Biophys Res Commun 355: 543-548, 2007.

26. Chang SC, Erwin AE and Lee AS: Glucose-regulated protein (GRP94 and GRP78) genes share common regulatory domains and are coordinately regulated by common trans-acting factors Mol Cell Biol 9: 2153-2162, 1989.
27. Dai L, Lou W, Zhu J, Zhou X and Di W: miR-199a inhibits the angiogenic potential of endometrial stromal cells under hypoxia by targeting HIF-1 $\alpha /$ VEGF pathway. Int J Clin Exp Pathol 8: 4735-4744, 2015

28. Guo H, Ingolia NT, Weissman JS and Bartel DP: Mammalian microRNAs predominantly act to decrease target mRNA levels. Nature 466: 835-840, 2010.

29. Nallamshetty S, Chan SY and Loscalzo J: Hypoxia: A master regulator of microRNA biogenesis and activity. Free Radic Biol Med 64: 20-30, 2013.

30. Haghikia A, Missol-Kolka E, Tsikas D, Venturini L, Brundiers S, Castoldi M, Muckenthaler MU, Eder M, Stapel B, Thum T, et al: Signal transducer and activator of transcription 3-mediated regulation of miR-199a-5p links cardiomyocyte and endothelial cell function in the heart: A key role for ubiquitin-conjugating enzymes. Eur Heart J 32: 1287-1297, 2011.

31. Gu Q, Kong Y, Yu ZB, Bai L and Xiao YB: Hypoxia-induced SOCS3 is limiting STAT3 phosphorylation and NF- $\kappa$ B activation in congenital heart disease. Biochimie 93: 909-920, 2011. 